\title{
Morcegos de Frederico Westphalen, Rio Grande do Sul, Brasil (Mammalia: Chiroptera): Riqueza e utilização de abrigos
}

\author{
Itiberê Piaia Bernardi ${ }^{1,2,8}$, João Marcelo Deliberador Miranda ${ }^{1,3}$, Jonas Sponchiado ${ }^{4}$, Eduardo Grotto $^{5}$, \\ Fábio Facco Jacomassa ${ }^{6}$, Eli Maria Teixeira ${ }^{5}$, Suelen Holterman Roani ${ }^{4}$ \& Fernando Camargo Passos ${ }^{1,7}$ \\ ${ }^{1}$ Laboratório de Biodiversidade, Conservação e Ecologia de Animais Silvestres, \\ Universidade Federal do Paraná - UFPR, CP 19020, CEP 81531-980, Curitiba, PR, Brasil \\ ${ }^{2} P P G$ em Ecologia e Conservação, Universidade Federal do Paraná - UFPR \\ ${ }^{3} P P G$ em Zoologia, Universidade Federal do Paraná - UFPR, \\ ${ }^{4}$ PPG em Biodiversidade Animal, Universidade Federal de Santa Maria - UFSM, Santa Maria, RS, Brasil \\ ${ }^{5}$ PPG em Biotecnologia e Gestão Ambiental, \\ Faculdade de Ciências Sociais Aplicadas, Xaxim, SC, Brasil \\ ${ }^{6} \mathrm{PPG}$ em Biologia, Universidade do Vale do Rio dos Sinos - UNISINOS, São Leopoldo, RS, Brasil \\ ${ }^{7}$ Departamento de Zoologia, Universidade Federal do Paraná - UFPR, Curitiba, PR, Brasil \\ ${ }^{8}$ Autor para correspondência: Itiberê Piaia Bernardi, e-mail: sturnira@gmail.com
}

BERNARDI, I.P., MIRANDA, J.M.D., SPONCHIADO, J., GROTTO, E., JACOMASSA, F.F., TEIXEIRA, E.M., ROANI, S.H. \& PASSOS, F.C. Bats of Frederico Westphalen, Rio Grande do Sul, Brazil (Mammalia: Chiroptera): Richness and shelter use. Biota Neotrop. 9(3): http://www.biotaneotropica.org.br/v9n3/en/abstr act?inventory+bn03009032009.

\begin{abstract}
The state of Rio Grande do Sul has, up to the present, records of 38 of the 168 bat species known to occur in Brazil, and several of its regions are severely understudied. In this study, a list of chiropteran species of the municipality of Frederico Westphalen, in the northernmost region of Rio Grande do Sul, is provided, as well as information on the use of human buildings and abandoned mines for seven of the 25 species recorded in the municipality. From October, 2004 to June, 2008, sampling was carried out in eleven areas of the municipality in order to survey the local chiropterofauna. The presence of species was recorded based on captures using mist nets, active searches in shelters, and specimens obtained from local residents. The recorded species belonged to 13 genera, of which 11 belonged to the family Vespertilionidae, whereas the families Molossidae and Phyllostomidae were represented by seven species each. The results obtained in the present study place the Upper Uruguay among the regions of highest species richness in Rio Grande do Sul.
\end{abstract}

Keywords: artificial shelters, Upper Uruguay, Chiroptera, Deciduous Seasonal Forest.

BERNARDI, I.P., MIRANDA, J.M.D., SPONCHIADO, J., GROTTO, E., JACOMASSA, F.F., TEIXEIRA, E.M., ROANI, S.H. \& PASSOS, F.C. Morcegos de Frederico Westphalen, Rio Grande do Sul, Brasil (Mammalia: Chiroptera): Riqueza e utilização de abrigos. Biota Neotrop. 9(3): http://www.biotaneotropica.org.br/v9n3/ pt/abstract?inventory+bn03009032009.

Resumo: O Estado do Rio Grande do Sul possui, até o momento, registros para 38 das 168 espécies de morcegos ocorrentes no Brasil e possui extensas áreas carentes de amostragem. Neste trabalho apresenta-se uma lista de espécies de quirópteros do município de Frederico Westphalen no extremo norte do Rio Grande do Sul e são fornecidas informações sobre a utilização de edificações humanas e minas abandonadas para sete das 25 espécies registradas no município. De outubro de 2004 a junho de 2008 esforços amostrais foram realizados em onze áreas no município visando inventariar a quiropterofauna local. A presença das espécies foi constatada a partir de capturas com auxílio de redes de neblina, busca ativa em refúgios, bem como, de exemplares obtidos junto a comunidade. As espécies pertencem a 13 gêneros, das quais a família Vespertilionidae é representada por 11 espécies, Molossidae por sete espécies e Phyllostomidae por sete espécies. Os resultados obtidos no presente trabalho colocam o Alto Uruguai entre as regiões com maior riqueza de quirópteros do Rio Grande do Sul.

Palavras-chave: abrigos artificiais, Alto Uruguai, Chiroptera, Floresta Estacional Decidual. 


\section{Introdução}

Das 168 espécies de quirópteros com ocorrência conhecida para o Brasil (Miranda et al. 2006, 2007, Reis et al. 2007), 38 possuem registros no Rio Grande do Sul (Silva 1985, 1994, González 2003, Pacheco \& Marques 2006, Weber et al. 2006, Bernardi et al. 2007, 2009, Pacheco et al. 2007). Apesar de o Rio Grande do Sul, a partir da década de 80 , ter recebido um incremento considerável no conhecimento quiropterológico, a grande maioria dos estudos esteve concentrada na porção leste e nordeste, restando extensas áreas do Estado que receberam pouco ou nenhum esforço amostral (Pacheco \& Marques 2006; Pacheco et al. 2007).

As informações sobre a quiropterofauna do extremo norte do Estado, porção originalmente coberta por vastas extensões da Floresta Estacional Decidual, ainda remontam aos esforços de Wallauer \& Albuquerque (1986) que apresentaram dados preliminares sobre a mastofauna do Parque Estadual do Turvo (PET). Foi somente há cerca de 20 anos depois do trabalho destes autores (Op. Cit. 1986) que a lista de espécies da região foi aumentada, com os registros de: (1) Eumops auripendulus Shaw, 1800 no ex-Parque Estadual de Nonoai (atualmente Reserva Indígena) (Trierveiler et al. 2002), (2) Noctilio leporinus Linnaeus, 1758 no PET (Silva et al. 2005) e (3) Molossops neglectus Willians \& Genoways, 1980 no município de Frederico Westphalen (Bernardi et al. 2007).

De acordo com Pacheco et al. (2007) a região do presente estudo é prioritária para a realização de esforços visando à conservação dos morcegos no Rio Grande do Sul. Assim sendo, o presente trabalho tem por objetivo apresentar uma lista das espécies de morcegos registradas no município de Frederico Westphalen no extremo norte do Estado do Rio Grande do Sul, Brasil além de fornecer dados sobre a utilização de abrigos artificiais e implicações metodológicas.

\section{Materiais e Métodos}

\section{1. Área de estudo}

Frederico Westphalen $\left(27^{\circ} 21^{\prime} \mathrm{S}\right.$ e $\left.53^{\circ} 23^{\prime} \mathrm{O}\right)$ localiza-se na região do Médio Alto Rio Uruguai, a $522 \mathrm{~m}$ de altitude, no Estado do Rio Grande do Sul. O clima da região é subtropical do tipo Cfa segundo a classificação de Köppen, onde a temperatura média anual é em torno $18{ }^{\circ} \mathrm{C}$, com máximas no verão podendo atingir $41^{\circ} \mathrm{C}$ e mínimas no inverno com valores inferiores a $0{ }^{\circ} \mathrm{C}$. A precipitação média anual é elevada, geralmente entre 1.800 e $2.100 \mathrm{~mm}$, bem distribuídos ao longo do ano (Bernardi et al. 2007).

Até a década de 1950 a região ainda era coberta por vastas extensões de Floresta Estacional Decidual, que ao longo do tempo foi sendo removida para dar espaço à ocupação e uso humano. Ainda, na década de 1980 praticamente toda a cobertura florestal do município havia sido alterada, restando um mosaico formado por pequenos fragmentos florestais (poucos com mais de 100 hectares), inseridos em uma matriz agricultural (caracterizada por pequenas propriedades rurais de subsistência familiar).

Frederico Westphalen está estrategicamente localizado dentro da triangulação dos três últimos grandes remanescentes florestais do extremo norte do Estado. A área localiza-se a cerca de $40 \mathrm{~km}$ a sudeste do Parque Estadual do Turvo (17.491 ha), $28 \mathrm{~km}$ a oeste da Área Indígena de Nonoai (17.000 ha) e $14 \mathrm{~km}$ a nordeste da Terra Indígena de Guarita (14.740 ha).

\section{Coleta dos dados}

Entre outubro de 2004 e junho de 2008, foram realizadas amostragens de quirópteros em onze áreas no município (Apêndice I). Três destas áreas receberam esforços sistematizados, onde cada uma recebeu uma noite completa de amostragem por mês entre outubro de 2005 e setembro de 2006, totalizando 36 noites de coletas ( 12 noites em cada área): Área I - fragmento florestal em zona rural com aproximadamente 35 hectares, localizado na localidade Vila Faguense, predominando vegetação em estágio secundário de sucessão. Essa vegetação caracteriza a mata ciliar do lajeado Tunas. Área II - fragmento periurbano com 45 hectares, também caracterizado por vegetação em estágio secundário de sucessão, localizado nas dependências da Universidade Regional Integrada do Alto Uruguai e das Missões (URI). Área III - bosque urbano, localizado nas dependências da Sociedade Aquática Barrilense, composto por áreas gramadas com vegetação esparsa, formada por espécies nativas e exóticas. O esforço de captura, para as três áreas, calculado de acordo com Straube \& Bianconi (2002) foi de 45,3 $\times 10^{3} \mathrm{~m}^{2} . \mathrm{h}$.

As demais áreas receberam esforços variados em número de noites, número de redes e horas de exposição. Com exceção da Área III (bosque urbano), as outras dez áreas consistem de fragmentos florestais com dimensões variando de 1 a 60 ha, e estão localizados entre 0 e $15 \mathrm{~km}$ da zona urbana do município.

Os morcegos foram capturados com redes de neblina $(7 \times 2,5 \mathrm{~m})$ posicionadas a $0,5 \mathrm{~m}$ do solo em trilhas, clareiras, bordas da mata, bem como perpendicularmente sobre cursos d'água. Para compor a lista de espécies, treze abrigos previamente selecionados devido à ocupação confirmada por morcegos foram visitados. Os morcegos foram capturados manualmente dentro dos abrigos, com redes de neblina e com armadilha tipo funil, posicionadas na saída do abrigo. Exemplares recebidos da comunidade e com procedência segura foram também incluídos.

A identificação em nível específico foi realizada sempre que possível ainda em condições de campo. Exemplares testemunho foram coletados, fixados e estão depositados na Coleção Científica de Mastozoologia do Departamento de Zoologia, Universidade Federal do Paraná (DZUP/CCMZ) (Apêndice II). As identificações foram realizadas de acordo com os critérios apresentados por LaVal (1973), Vizotto \& Taddei (1973), López-González (1998), Taddei et al. (1998), Barquez et al. (1999), López-González et al. (2001) e Gregorin \& Taddei (2002).

\section{Resultados e Discussão}

Foram registradas, até o momento, em Frederico Westphalen 25 espécies de morcegos pertencentes a três famílias, representando 65,7\% das espécies conhecidas para o Rio Grande do Sul (Tabela 1).

A família Vespertilionidae, representada por onze espécies, apresentou a maior riqueza, 44\%, seguida por Molossidae (28\%) e Phyllostomidae (28\%), ambas com sete espécies. A maior riqueza de Vespertilionidae em detrimento a Phyllostomidae contrasta com o observado em regiões de baixas latitudes e reflete a influência do clima temperado sobre as altas latitudes neotropicais de clima subtropical (Stevens 2004).

Das espécies listadas algumas merecem destaque devido a sua raridade ou pouca abundância no Rio Grande do Sul e/ou seu status de conservação (para espécies de difícil identificação são apresentadas informações adicionais):

Pygoderma bilabiatum (Wagner, 1843) - Espécie considerada rara no Rio Grande do Sul por Silva (1994) e listada como espécie com "dados insuficientes" por Pacheco \& Freitas (2003). Em Frederico Westphalen foi capturado nas áreas 1, 2, 3, 4, 5 e 7.

Platyrrhinus lineatus (E. Geoffroy, 1810) - De ocorrência presumível para o Rio Grande do Sul (Fabián et al. 1999) foi capturado no Estado apenas recentemente (Weber et al. 2006). Em Frederico 
Tabela 1. Lista de morcegos registrados no município de Frederico Westphalen, RS. I = Faguense; II = URI; III = SAB; Outra = espécie registrada em uma das demais áreas ou recebida de populares; Abrigo = espécie registrada utilizando edificações humanas ou minas abandonadas; $\bigcirc=$ noites sistematizadas; - = noites esporádicas; $\square=$ recebido de populares; $\mathrm{e} \bullet=$ presença da espécie na área.

Table 1. List of bats recorded in the municipality of Frederico Westphalen, RS. I = Faguense; II = URI, III = SAB; Other = species recorded in one of the other areas of popular or received; Shelter $=$ species recorded using human buildings or abandoned mines; $\circ=$ nights systematized; $\boldsymbol{\square}=$ sporadic nights; $\square=$ received the popular; and $\bullet=$ presence of species in the site.

\begin{tabular}{|c|c|c|c|c|c|c|}
\hline \multirow[t]{2}{*}{ Família/espécie } & \multirow[t]{2}{*}{ Tipo de registro } & \multicolumn{5}{|c|}{ Local de registro } \\
\hline & & I & II & III & Outra & Abrigo \\
\hline \multicolumn{7}{|l|}{ Phyllostomidae } \\
\hline Chrotopterus auritus (Peters, 1856) & $\circ$ & $\bullet$ & - & - & $\bullet$ & - \\
\hline Artibeus fimbriatus Gray, 1838 & $\circ$ & $\bullet$ & $\bullet$ & $\bullet$ & $\bullet$ & - \\
\hline A. lituratus (Olfers, 1818) & $\mathrm{Om}$ & $\bullet$ & $\bullet$ & $\bullet$ & $\bullet$ & - \\
\hline Sturnira lilium (É. Geoffroy, 1810) & $\odot \square$ & $\bullet$ & $\bullet$ & $\bullet$ & $\bullet$ & - \\
\hline Pygoderma bilabiatum (Wagner, 1843) & $\circ$ & $\bullet$ & $\bullet$ & $\bullet$ & $\bullet$ & - \\
\hline Platyrrhinus lineatus (É. Geoffroy, 1810) & $\circ$ & - & - & $\bullet$ & - & - \\
\hline Desmodus rotundus (É. Geoffroy, 1810) & $\square$ & - & - & - & $\bullet$ & $\bullet$ \\
\hline \multicolumn{7}{|l|}{ Vespertilionidae } \\
\hline Eptesicus furinalis (d'Orbigny \& Gervais, 1847) & - & $\bullet$ & $\bullet$ & $\bullet$ & $\bullet$ & $\bullet$ \\
\hline E. diminutus Osgood, 1915 & o & $\bullet$ & $\bullet$ & $\bullet$ & $\bullet$ & - \\
\hline E. brasiliensis (Desmarest, 1819) & - & - & - & - & $\bullet$ & - \\
\hline Myotis ruber (E. Geoffroy, 1806) & o曰 & $\bullet$ & - & - & - & - \\
\hline M. albescens (E. Geoffroy, 1806) & - & $\bullet$ & - & $\bullet$ & - & - \\
\hline M. nigricans (Schinz, 1821) & oш & $\bullet$ & $\bullet$ & - & - & - \\
\hline M. riparius Handley, 1960 & on & $\bullet$ & $\bullet$ & $\bullet$ & $\bullet$ & - \\
\hline Histiotus velatus (I. Geoffroy, 1824) & Om & $\bullet$ & $\bullet$ & $\bullet$ & $\bullet$ & $\bullet$ \\
\hline Lasiurus cinereus (Beauvois, 1796) & $\square$ & - & - & - & $\bullet$ & - \\
\hline L. blossevillii (Lesson \& Garnot, 1826) & $\square$ & $\bullet$ & - & - & $\bullet$ & - \\
\hline L. ega (Gervais, 1856) & $\square$ & - & - & - & $\bullet$ & - \\
\hline \multicolumn{7}{|l|}{ Molossidae } \\
\hline Molossus rufus É. Geoffroy, 1805 & $\square$ & - & - & - & $\bullet$ & $\bullet$ \\
\hline M. molossus (Pallas, 1766) & On & - & - & $\bullet$ & $\bullet$ & $\bullet$ \\
\hline Nyctinomops laticaudatus (É. Geoffroy, 1805) & $\square$ & - & - & - & $\bullet$ & - \\
\hline Tadarida brasiliensis (I. Geoffroy, 1824) & $\boldsymbol{\square} \square$ & $\bullet$ & - & - & $\bullet$ & - \\
\hline Molossops neglectus Willians \& Genoways, 1980 & - & $\bullet$ & $\bullet$ & - & $\bullet$ & - \\
\hline Promops nasutus (Spix, 1823) & $\square$ & - & - & - & $\bullet$ & - \\
\hline Eumops bonariensis (Peters, 1874) & $\square$ & - & - & - & $\bullet$ & - \\
\hline \multirow[t]{2}{*}{ Total } & & 15 & 10 & 11 & 21 & 5 \\
\hline & & & & 25 & & \\
\hline
\end{tabular}

Westphalen um único exemplar foi capturado até o momento na área 3.

Eptesicus diminutus Osgood, 1915 - Silva (1994) considera a espécie rara para o Estado. Em Frederico Westphalen foi capturada nas áreas 1, 2, 3, 4, 5 e 7. Os exemplares aqui atribuídos a E. diminutus apresentaram o conjunto de caracteres diagnóstico da espécie de acordo com Barquez et al. (1999), a constar: calcâneo maior do que o dobro do comprimento do pé, ponta das orelhas mais arredondadas e lobo basal do trago menos desenvolvido quando comparado a $E$. furinalis. Todos os exemplares examinados apresentaram comprimento do antebraço entre 34,0 e $36,0 \mathrm{~mm}$ e comprimento da mandíbula menor do que $10,1 \mathrm{~mm}$.

Myotis ruber (E. Geoffroy, 1806) - Considerada rara por Silva (1984) e ameaçada de extinção sob a categoria "Vulnerável" no Rio Grande do Sul por Pacheco \& Freitas (2003). A espécie foi capturada apenas na área 1 .
Myotis albescens (E. Geoffroy, 1806) - Listada como espécie com "dados insuficientes" por Pacheco \& Freitas (2003), teve recentemente reavaliada sua distribuição geográfica no Rio Grande do Sul, estando presente em oito localidades (Quintela et al. 2008). Em Frederico Westphalen a espécie foi capturada nas áreas 1 e 3. Os exemplares aqui atribuídos a $M$. albescens foram identificados de acordo com LaVal (1973), Barquez et al. (1999), López-González (1998), López-González et al. (2001). Como caracteres diagnósticos os espécimes examinados apresentam largura da constrição pósorbitária maior do que 4,0 mm, comprimento do antebraço entre 36,0 e $37,0 \mathrm{~mm}$, rostro relativamente curto quando comparado a M. levis, série de dentes inferiores menor do que 5,6 $\mathrm{mm}$, ventre esbranquiçado (especialmente na região perianal e nas laterais do corpo) e uropatágio com a borda pigmentada e apresentando uma franja de pelos.

Myotis riparius Handley, 1960 - Categorizada como "dados insuficientes" por Pacheco \& Freitas (2003), com registros apenas 
para Santana do Livramento e Caxias do Sul a espécie tem agora sua distribuição ampliada até o extremo norte do estado. Em Frederico Westphalen $M$. riparius foi capturada nas áreas 1, 2, 3, 4, 5 e 7.

Nyctinomops laticaudatus (E. Geoffroy, 1805) - Listada como espécie com "dados insuficientes" por Pacheco \& Freitas (2003), possui ocorrência conhecida para cinco localidades no Estado: Rio Grande, Santa Vitória do Palmar, Porto Alegre, Torres e Santa Maria (Pacheco \& Freitas, 2003; Weber et al. 2007). O presente trabalho aumenta para seis os municípios com registros desta espécie no Rio Grande do Sul. Em Frederico Westphalen, N. laticaudatus foi encontrado pela comunidade no interior de uma residência na área urbana do município.

Molossops neglectus Willians \& Genoways, 1980 - Recentemente registrado pela primeira vez no Estado (Bernardi et al. 2007), a espécie tem sido capturada tanto na área rural como na urbana do município nas áreas 1, 2 e 4. Frederico Westphalen é o atual limite sul na distribuição desta espécie (Bernardi et al. 2007).

Eumops bonariensis (Peters, 1874) - Listada como espécie com “dados insuficientes" por Pacheco \& Freitas (2003), E. bonariensis foi capturado em uma única oportunidade no interior de uma residência na área urbana do município. A identificação do espécime em questão seguiu os critérios apresentados em Sanborn (1932), Eger (1977), López-González (1998), Barquez et al. (1999), Gregorin \& Taddei (2002) e Bernardi et al. (2009).

Das 25 espécies registradas, sete foram observadas utilizando edificações humanas como abrigo e uma, Desmodus rotundus (E. Geoffroy, 1810), utilizando minas de extração de ametista abandonadas próximas a área 10.

Os treze abrigos visitados puderam ser separados em sete modalidades diferentes: vão de dilatação $(n=4)$, forro (sótão) $(n=4)$, garagem $(n=2)$, porão $(n=1)$, parede dupla $(n=1)$, prédio em construção $(\mathrm{n}=1)$ e mina abandonada $(\mathrm{n}=1)$. Histiotus velatus (I. Geoffroy, 1824) foi a espécie mais observada, utilizando cinco modalidades diferentes de abrigos: vão de dilatação $(n=2)$; garagem $(\mathrm{n}=2)$; prédio em construção $(\mathrm{n}=1)$; forro $(\mathrm{n}=1)$ e porão $(\mathrm{n}=1)$; Molossus molossus (Pallas, 1766), foi observado em vão de dilatação $(\mathrm{n}=1)$ e porão $(\mathrm{n}=1)$. Molossus rufus E. Geoffroy, 1805 usa forro $(\mathrm{n}=1)$ e parede dupla $(\mathrm{n}=1)$; Tadarida brasiliensis (I. Geoffroy, 1824), forro $(\mathrm{n}=1)$ e porão $(\mathrm{n}=1)$; Eptesicus furinalis (d'Orbigny \& Gervais, 1847) em vão de dilatação $(n=2)$. Observou-se coabitação interespecífica em três oportunidades: o trio $T$. brasiliensis, $H$. velatus e M. molossus em (1) forro e em (2) porão; E. furinalis e M. molossus em (3) vão de dilatação. Sturnira lilium (E. Geoffroy, 1810) $(\mathrm{n}=1) \mathrm{e}$ Myotis albescens $(\mathrm{n}=1)$ foram capturados no interior do porão com rede de neblina, no entanto, não foram observados indivíduos pousados nem agrupamentos destas espécies. Estes exemplares poderiam estar utilizando o abrigo apenas ocasionalmente já que o momento da captura foi durante o início de uma forte chuva.

Com base nos resultados apresentados na Tabela 1 fica evidente a importância da combinação de métodos amostrais quando a riqueza de espécies é parte dos objetivos do estudo. Mediante o uso de redes de neblina (excluindo-se o uso intencional na saída de abrigos) foram registradas 17 espécies e por meio de buscas em abrigos (incluindo captura manual, puçás, redes de neblina e armadilha de funil) foram registradas oito espécies.

Cabe ressaltar que exemplares de 13 espécies foram recebidos por populares e destas, cinco foram registradas exclusivamente por meio de doações (Lasiurus cinereus (Beauvois, 1796), L. ega (Gervais, 1856), Nyctinomops laticaudatus, Promops nasutus (Spix, 1823) e Eumops bonariensis). Estes dados corroboram com os apresentados por Brosset et al. (1996) de que as espécies insetívoras pertencentes às famílias Vespertilionidae e Molossidae são mais dificilmente capturadas pelos métodos tradicionais como por exemplo as redes de neblina.

Os dados obtidos colocam o Alto Uruguai entre as regiões com maior riqueza de quirópteros do Estado, comparável às regiões leste e nordeste (ver Pacheco \& Marques, 2006, Pacheco et al. 2007) e sugerem que a riqueza de morcegos do Parque Estadual do Turvo, com apenas nove espécies, pode estar sendo subestimada.

As informações aqui apresentadas contribuem substancialmente para o conhecimento da fauna de morcegos do norte do Estado, principalmente no que tange aos aspectos distribucionais, evidenciando a necessidade de inventários em áreas ainda não amostradas bem como a continuidade dos esforços em áreas já amostradas, buscando uma melhor caracterização da quiropterofauna gaúcha.

\section{Agradecimentos}

Os autores agradecem Fernando Carvalho, Janaina Gazarini e dois revisores anônimos pela leitura crítica e sugestões aos originais. Aos proprietários e responsáveis pelas áreas amostradas por permitirem a realização do trabalho. Ao CNPq pela bolsa concedida a JMDM e FCP. Ao Instituto Brasileiro do Meio Ambiente e dos Recursos Naturais Renováveis, IBAMA pelas licenças 048/2006 e 10300-1.

\section{Referências Bibliográficas}

BARQUEZ, R.M., MARES, M.A. \& BRAUN, J.K. 1999. The bats of Argentina. Museum of Texas Tech University, Lubbock, p. 1-275. (publicação especial, n. 42).

BERNARDI, I.P., PULCHÉRIO-LEITE, A., IRANDA, J.M.D. \& PASSOS, F.C. 2007. Ampliação da distribuição de Molossops neglectus Williams e Genoways (Chiroptera, Molossidae) para o Sul da América do Sul. Rev. Bras. Zool. 24(2):505-507.

BERNARDI, I.P., MIRANDA, J.M.D. \& PASSOS, F.C. 2009. Status taxonômico e distribucional do complexo Eumops bonariensis no Sul do Brasil. Zoologia. 26(1):183-190.

BROSSET, A., CHARLES-DOMINIQUE, P., COCKLE, A., COSSON, J.F. \& MASSON, D. 1996. Bat communities and deforestation in French Guiana. Can. J. Zool. 74:1974-1982.

EGER, J.L. 1977. Systematics of the genus Eumops (Chiroptera: Molossidae). Royal Ontario Museum, Toronto, p. 1-69. (Life Sciences Contributions, n. 110).

FABIÁN, M.E., RUI, A.M. \& OLIVEIRA, K.P. 1999. Distribuição geográfica de morcegos Phyllostomidae (Mammalia: Chiroptera) no Rio Grande do Sul, Brasil. Iheringia, Zool. 87:143-156.

GREGORIN, R. \& TADDEI, V.A. 2002. Chave artificial para a identificação de Molossídeos brasileiros (Mammalia, Chiroptera). Mastozool. neotrop. 9(1):13-32.

GONZÁLEZ, J.C. 2003. Primeiro registro de Eumops patagonicus Thomas, 1924 para o Brasil (Mammalia: Chiroptera: Molossidae). Comun. Mus. Cienc. Tecnol. PUCRS, Ser. zool. 16(2):255-258.

LAVAL, R.K. 1973. A revision o the Neotropical bats of genus Myotis. Nat. His. Mus. Los Angeles County Sci. Bull. 15:1-54.

LÓPEZ-GONZÁLEZ, C. 1998. Systematics and biogeography of the bats of Paraguay. Tese de Doutorado, Texas Tech University, Lubbock, 395 p.

LÓPEZ-GONZÁLEZ, C., PRESLEY, S.J., OWEN, R.D. \& WILLIG, M.R. 2001. Taxonomic status of Myotis (Chiroptera: Vespertilionidae) in Paraguay. J. Mammal. 82(1):138-160.

MIRANDA, J.M.D., BERNARDI, I.P. \& PASSOS, F.C. 2006. A new species of Eptesicus (Mammalia: Chiroptera: Vespertilionidae) from Atlantic Forest Brazil. Zootaxa. 1383:57-68.

MIRANDA, J.M.D., AZEVEDO-BARROS, M.F.M. \& PASSOS, F.C. 2007. First record of Histiotus laephotis Thomas (Chiroptera, Vespertilionidae) from Brazil. Rev. Bras. Zool. 24(4): 1188-1191. 
PACHECO, S.M. \& FREITAS, T.R.O. 2003. Quirópteros. In Livro vermelho da fauna ameaçada de extinção no Rio Grande do Sul. (C.S. Fontana, G.A. Bencke, \& R.E. Reis, eds). EDIPUCRS, Porto Alegre, p. 483-497.

PACHECO, S.M. \& MARQUES, R.V. 2006. Conservação de morcegos no Rio Grande do Sul. In Mamíferos do Brasil: genética, sistemática, ecologia e conservação (T.R.O. Freitas, E. Vieira, S.M. Pacheco \& A. Christoff, eds). Suprema, São Carlos, p. 91-106.

PACHECO, S.M., SEKIAMA, M.L., OLIVEIRA, K.P.A., QUINTELA, F., WEBER, M.M., MARQUES, R.V., GEIGER, D. \& SILVEIRA, D.D. 2007. Biogeografia de quirópteros da Região Sul. Ciência e Ambiente. $35: 181-202$.

QUINTELA, F.M., PORCIÚNCULA, R.A. \& PACHECO, S.M. 2008. Mammalia, Chiroptera, Vespertilionidae, Myotis albescens: new occurrence site in the state of Rio Grande do Sul, Brazil. Check List. 4(1):79-81.

REIS, N.R., PERACCHI, A.L., PEDRO, W.A. \& LIMA, I.P. 2007. Morcegos do Brasil. Editora da Universidade Estadual de Londrina, Londrina, $253 \mathrm{p}$.

SANBORN, C.C. 1932. The bats of the genus Eumops. J. Mammal. 13:347-357.

SILVA, C.P., MÄHLER Jr., J.K.F., MARCUZZO, S.B. \& FERREIRA, S. 2005. Plano de manejo do Parque Estadual do Turvo. Secretaria Estadual de Meio Ambiente, Porto Alegre, 355 p.

SILVA, F. 1985. Guia para determinação de morcegos: Rio Grande do Sul. Martins Livreiro, Porto Alegre, $77 \mathrm{p}$.

SILVA, F. 1994. Mamíferos silvestres: Rio Grande do Sul. Fundação Zoobotânica do Rio Grande do Sul, Porto Alegre, 246 p. (Publicações Avulsas FZB, n. 7).
STEVENS, R.D. 2004. Untangling latitudinal richness gradients at higher taxonomic levels: familial perspectives on the diversity of New World bat communities. J. Biogeogr. 31:665-674.

STRAUBE, F.C. \& BIANCONI, G. 2002. Sobre a grandeza e a unidade utilizada para estimar esforço de captura com utilização de redes-deneblina. Chiropt. neotrop. 8(1/2):150-152.

TADDEI, V.A., NOBILE, C.A. \& MORIELLE-VERSUTE, E. 1998. Distribuição geográfica e análise morfométrica comparativa em Artibeus obscurus (Schinz, 1821) e Artibeus fimbriatus Gray, 1838 (Mammalia, Chiroptera, Phyllostomidae). Ens. cienc. 2(2):71-127.

TRIERVEILER, F., ANDRADE, F.M. \& FREITAS, T.R.O. 2002. Karyotype of Eumops auripendulus major (Chiroptera: Molossidae) and its first recorded sighting in southern Brazil. Mammalia. 66:303-306.

VIZOTTO, L.D. \& TADDEI, V.A. 1973. Chave para determinação de quirópteros brasileiros. Boletim de Ciências, Faculdade de Filosofia, Ciências e Letras. 1:1-72.

WALLAUER, J.P. \& ALBUQUERUQE, E.P. 1986. Lista preliminar dos mamíferos observados no Parque Florestal Estadual do Turvo, Tenente Portela, RS, Brasil. Roessléria. 8(2):179-185.

WEBER, M.M., ARRUDA, A.J. \& CÁCERES, N.C. 2007. Ampliação da distribuição de quatro espécies de morcegos (Mammalia, Chiroptera) no Rio Grande do Sul, Brasil. Biota neotrop.7(2): 293-296.

WEBER, M.M., CÁCERES, N.C., LIMA, D.O., CAMILOTTI, V.L., ROMAN, C. \& NETO, L.T. 2006. Mammalia, Chiroptera, Phyllostomidae, Platyrrhinus lineatus: range expansion to the state of Rio Grande do Sul, Brazil. Check List. 2(3):96-98.

Recebido em 19/05/09 Versão reformulada recebida em 06/07/09 Publicado em 10/07/09 
Bernardi, I.P. et al.

\section{Apêndice I}

Lista de topônimos e suas respectivas coordenadas geográficas e altitudes:

1 - Vila Faguense, $27^{\circ} 22^{\prime} 28^{\prime}$ 'S e $53^{\circ} 25^{\prime} 37^{\prime}$ 'W, $471 \mathrm{~m}$;

2 - Universidade Regional Integrada do Alto Uruguai e das Missões, $27^{\circ} 21^{\prime}$ 42” S e 532 24' 37' W, 556 m;

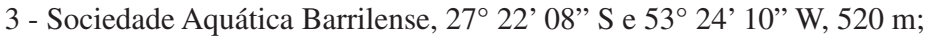

4 - Santuário de Schoenstatt, $27^{\circ} 22^{\prime} 39^{\prime \prime}$ S e 52 24' 142” W, 558 m;

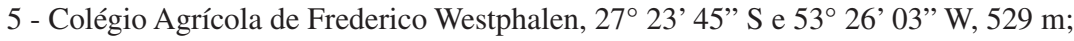

6 - Linha Boa Esperança A, $27^{\circ} 20^{\prime} 45^{\prime}$ 'S e 5324' 57' W, 515 m;

7 - Linha Boa Esperança B, $27^{\circ} 21^{\prime} 12^{\prime \prime} \mathrm{S}$ e $53^{\circ} 26^{\prime} 06^{\prime \prime} \mathrm{W}, 451 \mathrm{~m}$;

8 - Linha Pavão, $27^{\circ} 17^{\prime} 20^{\prime \prime}$ S e 5329' 31' W, 284 m;

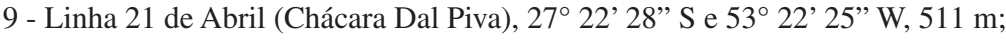

10 - Castelinho/Rio da Várzea, $27^{\circ} 20^{\prime} 49^{\prime \prime}$ S e 53 $3^{\circ} 16^{\prime} 02^{\prime \prime} \mathrm{W}, 225 \mathrm{~m}$; e

11 - Castelinho/Garimpo, $27^{\circ} 21^{\prime} 53^{\prime \prime} \mathrm{S}$ e $53^{\circ} 15^{\prime} 59^{\prime \prime} \mathrm{W}, 361 \mathrm{~m}$.

\section{Apêndice II}

Exemplares depositados $(n=74)$ na Coleção Científica de Mastozoologia do Departamento de Zoologia da Universidade Federal do Paraná (DZUP/CCMZ) e seus respectivos números tombo.

Chrotopterus auritus 684,685; Artibeus fimbriatus 686,687; A. lituratus 688,689,690, 691,692,693; Sturnira lilium 49, 60, 281, 182, 283, 284, 285, 286, 287, 288; Pygoderma bilabiatum 339; Platyrrhinus lineatus 694; Desmodus rotundus 695; Eptesicus furinalis 663, 668, 670, 678; E. diminutus 664, 665, 667, 671, 679, 681; E. brasiliensis 682; Myotis ruber 696, 697; M. albescens 574, 575, 576; M. nigricans 338, 674; M. riparius 577, 578, 672, 676; Histiotus velatus 656, 654, 655, 657; Lasiurus cinereus 659; L. blossevillii 658; L. ega 698,699; Molossus rufus 238, 239, 240, 241, 242, 243, 244; M. molossus 571, 572, 573; Nyctinomops laticaudatus 570; Tadarida brasiliensis 569; Molossops neglectus 220, 274, 275, 565, 566, 567, 568; Promops nasutus 670; Eumops bonariensis 526. 